\title{
Prenatal diagnosis of the isodicentric chromosome 22 associated with cat eye syndrome by multiplex ligation-dependent probe amplification
}

\author{
Sang Hee Park', Sung Han Shim', Yong Wook Jung², So Hyun Shim², Mi Uk Chin', Ji Eun Park', Sung Mi Bae', Sang Woo Lyu², and \\ Dong Hyun Cha ${ }^{1,2, *}$ \\ 'Genetic Laboratory, Fertility Center of CHA Gangnam Medical Center, CHA University, Seoul, Korea \\ ${ }^{2}$ Department of Obstetrics and Gynecology, CHA Gangnam Medical Center, CHA University, Seoul, Korea
}

Cat eye syndrome (CES) is a very rare chromosomal syndrome characterized by various malformations such as anal atresia, preauricular malformation, coloboma of the iris, and congenial heart and renal defects. This genetic disorder is caused by partial duplication of chromosome 22, mostly as a result of a supernumerary isodicentric marker chromosome idic(22)(q11.2). Various congenital abnormalities and extreme phenotypic variability in CES patients have been reported, which have made prenatal diagnosis of CES difficult. We report the first case diagnosed with CES prenatally by multiplex ligation-dependent probe amplification in a woman who was referred to our hospital, for a fetus presenting with heart anomaly.

Key words: Schmid-Fraccaro syndrome, Prenatal diagnosis of cat eye syndrome, Chromosome 22 partial tetrasomy, Multiplex polymerase chain reaction, Chromosome marker.

\section{Introduction}

Cat eye syndrome (CES), or Schmid-Fraccaro syndrome, is a rare condition caused by a small segment of chromosome $22 q 11.2$ being present in three (trisomic) or sometimes four copies (tetrasomic) (usually three copies) instead of the usual two copies. A supernumerary, bisatellited, and isodicentric marker chromosome is the most common form. The major clinical features of CES comprise of preauricular tags and/or pits, anorectal malformations, ocular colobomata, congenital heart defects, and mild to moderate mental retardation [1]. However, several phenotypes, ranging from normality to full CES, have been reported [2]. The incidence of CES has been estimated to be be- tween 1:50,000 and 1:150,000 in newborns [3].

Two types of CES have been classified on the basis of location of the two breakpoints. The smaller type 1 CES chromosomes have two proximal breakpoints without the DiGeorge critical region (DGCR). The larger type II CES chromosomes have one or two distal breakpoints, which contain one or two additional copies of the DGCR [4]. Since estimation of the direct correlation between the extent of duplicated chromosome 22 segment and the severity of the CES remains difficult, determination of the type of CES chromosome does not currently have prognostic value [5].

In this report, we present the first prenatal diagnosis of CES in a fetus showing heart anomaly. The combination of cytogenetic

\footnotetext{
Received: 9 June 2017, Revised: 24 June 2017, Accepted: 24 June 2017, Published: 30 June 2017

*Corresponding author: Dong Hyun Cha, M.D., Ph.D.

Department of Obstetrics and Gynecology, CHA Gangnam Medical Center, CHA University, 566 Nonhyun-ro, Gangnam-gu, Seoul 06135, Korea.

Tel: +82-2-3468-3132, Fax: +82-2-3468-2616, E-mail: chadh001@chamc.co.kr

Conflict of interest: The authors declare that they do not have any conflicts of interest.

(c) This is an open-access article distributed under the terms of the Creative Commons Attribution Non-Commercial License (http://creativecommons.org/licenses/by-nc/4.0/) which permits unrestricted non-commercial use, distribution, and reproduction in any medium, provided the original work is properly cited.

(c) Copyright 2017 by the Korean Society of Medical Genetics and Genomics 
and molecular genetic analyses including multiplex ligationdependent probe amplification (MLPA) is very useful to identify the origin of the supernumerary marker chromosome (SMC).

\section{Case}

A 37-year-old woman, gravida 3, para 0, ectopic pregnancy 2, was referred to the genetics laboratory of CHA Gangnam Medical Center at 22 weeks of gestation, for a fetus presenting with heart anomalies of type II interruption of aortic arch and large ventricular septal defect in fetal echocardiogram. An amniocentesis was performed for fetal karyotype determination. The cytogenetic analysis of 20 cells showed a SMC with a bisatellited and isodicentric form (Fig. 1). Both parents showed normal karyotypes, indicating that the marker chromosome was de novo. This marker chromosome was analyzed further by MLPA with P070-subtelomere and P245-microdeletion probemix (MRC-Holland, Amsterdam, the Netherlands). In results of MLPA analysis, duplication at 22q11.1 was detected by P070-subtelomere probemix (Fig. 2A) but not P245-microdeletion probemix (Fig. 2B) contained 22q11.21 region associated with DiGeorge syndrome. The duplication was located in the 22q11 CES region (IL17RA gene) in the fetus. Fluorescence in situ hybridization (FISH) analysis of this marker chromosome was conducted on metaphase spreads with a probe for the DGCR N25 (Vysis, Abbott Park, IL, USA), located on 22q11.2, which did not show any signal on the marker chromosome (Fig. 3). MLPA and FISH analyses concluded that the marker was a typical type 1 CES chromosome. Therefore, the karyotype of the fetus was defined as 47, XX + mar.rsa 22q11.1(IL17RA)X3 dn, resulting in trisomy of the proximal part of $22 q 11$.

\section{Discussion}

CES is a rare chromosomal syndrome characterized by duplication of the region that spans the chromosome 22p arm and part of 22q11, usually in the form of a bisatellited, isodicentric supernumerary chromosome [1]. The formation of the idic(22) of CES can be explained as mainly due to homologous recombination during meiosis, or breakage and reunion of the sister chromatids near the centromere [6].

The CES critical region (CESCR) has breakpoints between proximal locus ATP6E and distal locus D22S57, covering approximately $2 \mathrm{Mb}$ of 22q11.2 [7]. Fourteen genes have thus far been identified in the CESCR, and two of these genes, CECR 1 and CECR2 may be critical dosage-sensitive genes $[4,8]$.

Extreme phenotypic variability is particularly problematic as a de novo SMC(22) ascertained prenatally. In the first prenatal case report of CES, Volpe et al. [9] documented increased nuchal translucency, and ear and cardiac anomalies in fetal ultrasonographic investigation. Postnatal examination showed ocular colobomata, an imperforate anus, and facial dysmorphism.

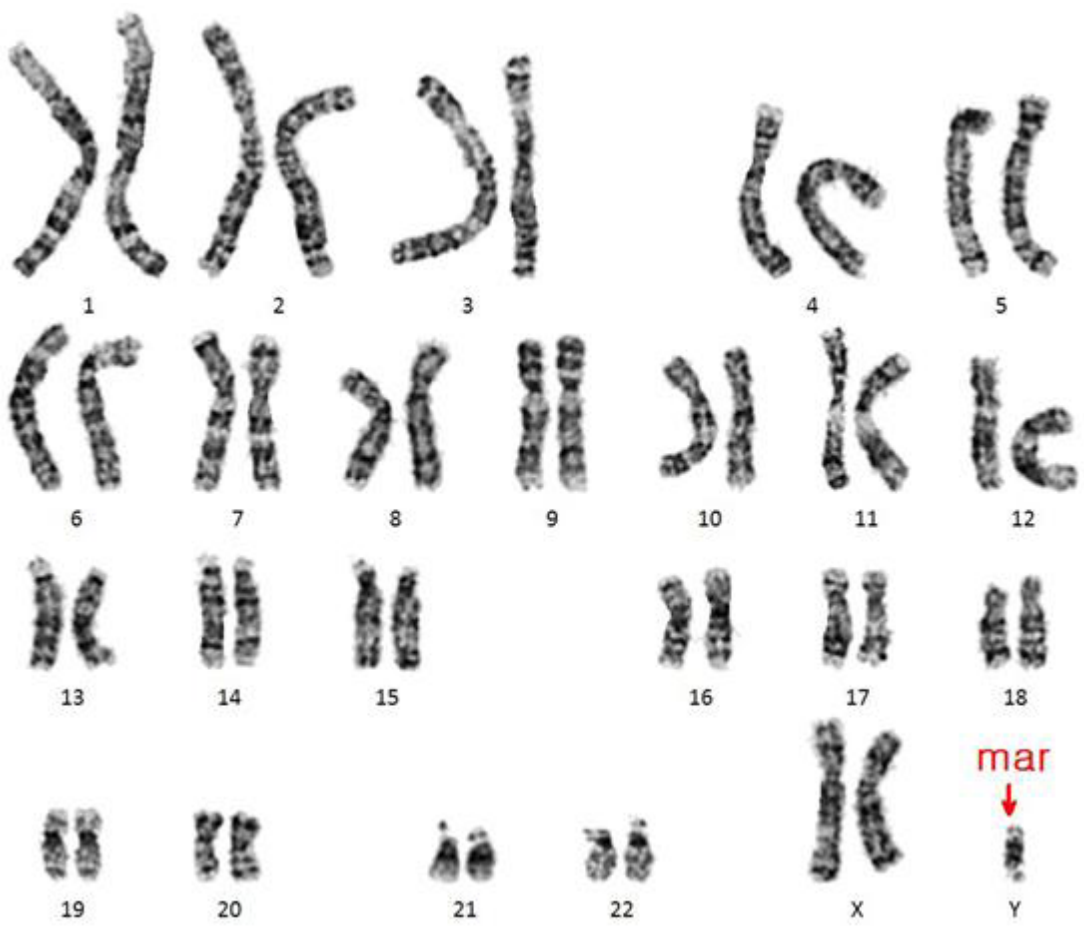

Fig. 1. GTG-banded metaphase chromosomes showing a supernumerary bisatellited marker. 
Rosias et al. [2] have reviewed the findings of previous reports with clinical features of 105 reported CES patients. They described the large phenotypic variability, ranging from marginally affected to full pattern of malformation, and lethal outcome. Most of the prenatal CES patients were diagnosed incidentally by fetal chromosome analysis referred for advanced maternal age but did not show any feature in ultrasonographic screening. It is difficult to reveal the features of CES such as iris coloboma and few other subtle features in the ultrasound screening at mid trimester [10]. Among CES malformations, preauricular skin tags and/or pits were the most consistent features. Berends et al. [11] studied a group of 74 patients and found that preauricular anomalies accounted for $81 \%$ of the CES patients. Evaluation of the fetal face, particularly the 'ear', is an important aspect of the mid trimester anomaly scan in prenatal screening that gives some important clues for either a syndromic or nonsyndromic
A
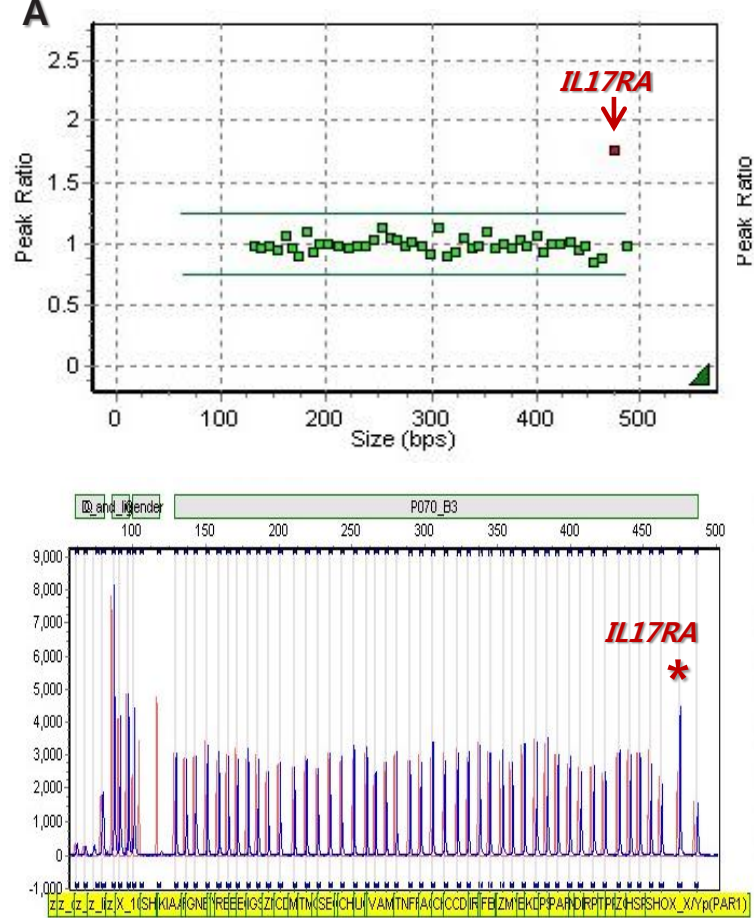
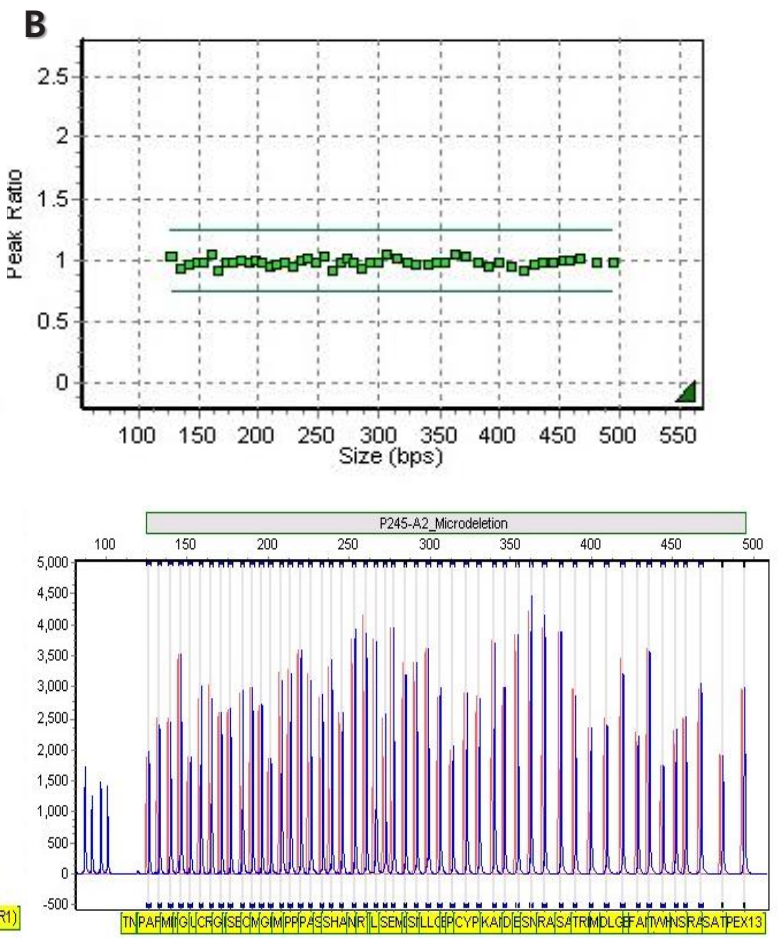

Fig. 2. MLPA (Multiplex ligation-dependent probe amplification) analysis using P070-subtelomere (A) and P245-microdeletion (B) probemix, respectively. IL17RA: Interleukin 17 receptor A.

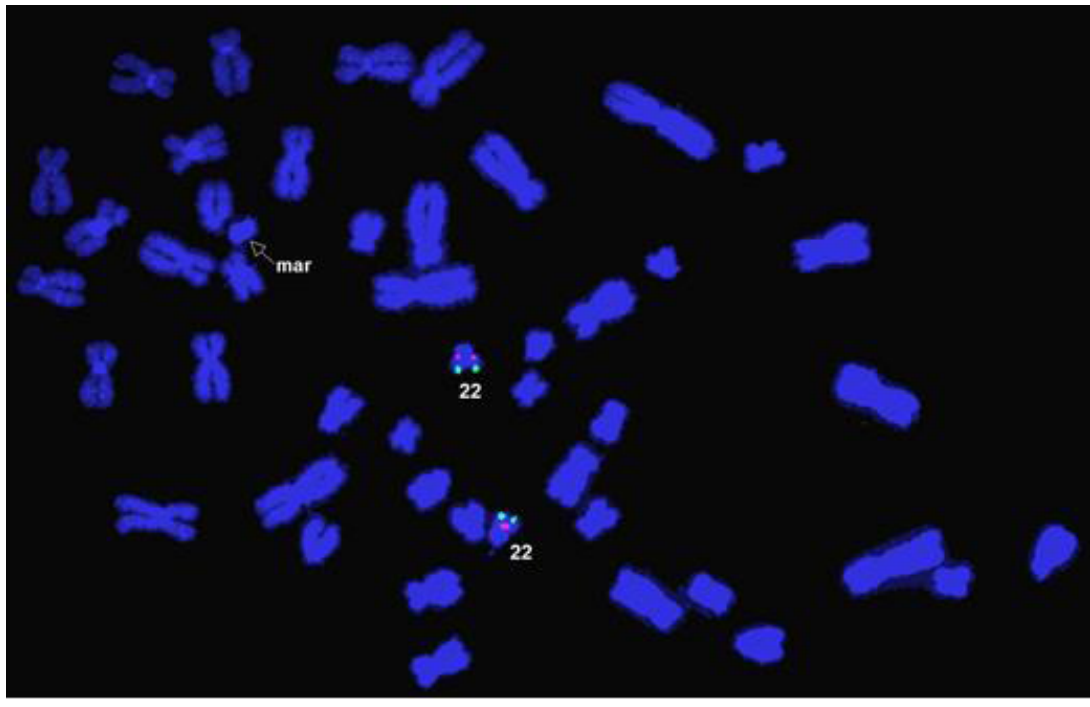

Fig. 3. Fluorescence in situ hybridization result with DiGeorge syndrome region (N25) probe. N25 and ARSA signals are absent in the marker (arrow) and present in the two normal chromosome 22. 
CES. The use of three- and four-dimensional ultrasonography enables easier and more rapid detection of ear malformations $[9,12]$. A protocol to investigate ear anomalies has been established for the prenatal diagnosis of chromosomal abnormalities [13].

Approximately $20 \%$ of SMCs are familial and are usually inherited without phenotypic effects [12]. In contrast, most of the characteristics related to CES are variably expressed even in familial cases. A previous report presented a familial supernumerary ring chromosome 22 in which the affected proband had four copies of CESCR, whereas the unaffected father and grandfather had three copies. They suggested a threshold model that the presence of four rather than three copies of this region may increase the susceptibility of an individual to express the CES malformations [14]. However, several studies reported that patients with three or four copies of 22q11.1-q11.2 have common malformations of CES ranging from coloboma of the iris, anal atresia, and craniofacial defects, suggesting that partial trisomy is enough to show the various malformations of CES $[7,15]$.

The correlations between karyotype and phenotype have been studied in patients with SMC(15)s. Patients with SMC(15)s containing additional copies of the proximal $15 q$ imprinted PraderWilli or Angelman syndrome critical regions have moderate to severe mental retardation. Parental origin studies revealed that the de novo SMC(15)s were of maternal origin, indicating that a parental origin of specific dosage imbalance is a causative factor in the phenotype. A similar mechanism accounting for phenotypic differences in CES patients seems unlikely because there is no report as yet of imprinting on chromosome 22. Further studies are required to determine whether parental origin of the CES marker has a specific dosage effect $[5,14]$.

In addition, the clinical severity of a CES patient does not seem to correlate with the size of the marker chromosome [5]. In our case, the fetus had a smaller type 1 CES chromosome without DGCR, but had a severe congenital heart anomaly. However, additional case studies are necessary to draw more specific genotype-phenotype correlations and to determine the outcome of patients with CES, especially when this rare condition is diagnosed prenatally [12].

Here, we report a prenatal CES diagnosis, which showed partial trisomy of chromosome 22q11.1 resulting from a supernumerary isodicentric marker chromosome. It is the first prenatal case of CES in Korea. Cytogenetic and molecular genetic analyses led to the confirmative diagnosis by identifying the origin of the marker chromosome. MLPA could rapidly and efficiently detect 22q11 duplication, and determine the type of CES chro- mosome. It is an economical, fast, and accurate method for the prenatal genetic diagnosis of the CES syndrome [16].

\section{References}

1. Chen H. Atlas of genetic diagnosis and counseling. New York: Springer, 2012.

2. Rosias PR, Sijstermans JM, Theunissen PM, Pulles-Heintzberger CF, De Die-Smulders CE, Engelen JJ, et al. Phenotypic variability of the cat eye syndrome. Case report and review of the literature. Genet Couns $2001 ; 12: 273-82$

3. Jedraszak G, Receveur A, Andrieux J, Naepels P, Mathieu-Dramard M, Bremond-Gignac $D$, et al. A severe prenatal presentation of Cat Eye Syndrome. Clin Dysmorphol 2013;22:175-7.

4. Ko JM, Kim JB, Pai KS, Yun JN, Park SJ. Partial tetrasomy of chromosome $22 q 11.1$ resulting from a supernumerary isodicentric marker chromosome in a boy with cat-eye syndrome. J Korean Med Sci 2010;25:1798-801.

5. Mears A, Duncan AM, Budarf ML, Emanuel BS, Sellinger B, SiegelBartelt J, et al. Molecular characterization of the marker chromosome associated with cat eye syndrome. Am J Hum Genet 1994;55:134-42.

6. Oruganti VP, Vidyadhari M, Buddhavarapu P, Kandukuri LR. Fetal loss: a genetic insight of the de novo accessory Bi-Satellited marker of chromosome 22P. J Genet Syndr Ther 2015;6:259.

7. Mears AJ, el-Shanti H, Murray JC, McDermid HE, Patil SR. Minute supernumerary ring chromosome 22 associated with cat eye syndrome: further delineation of the critical region. Am J Hum Genet 1995:57:667-73.

8. Quintero-Rivera F, Martinez-Agosto JA. Hemifacial microsomia in cat-eye syndrome: 22q11.1-q11.21 as candidate loci for facial symmetry. Am J Med Genet A 2013;161A:1985-91.

9. Volpe P, Buonadonna AL, Campobasso G, Di Carlo A, Stanziano A, Gentile M. Cat-eye syndrome in a fetus with increased nuchal translucency: three-dimensional ultrasound and echocardiographic evaluation of the fetal phenotype. Ultrasound Obstet Gynecol 2004;24:485-7.

10. Huang B, Solomon S, Thangavelu M, Peters K, Bhatt S. Supernumerary marker chromosomes detected in 100,000 prenatal diagnoses: molecular cytogenetic studies and clinical significance. Prenat Diagn 2006;26:1142-50.

11. Berends MJ, Tan-Sindhunata G, Leegte B, van Essen AJ. Phenotypic variability of Cat-Eye syndrome. Genet Couns 2001;12:23-34.

12. Royal ABP, Hegde $S$, Radhakrishnan P, Prathima R, Jayaprakash. Importance of ear and face abnormalities in cat eye syndrome - A prenatal and postnatal report. Eur J Biotechnol Bioscience 2014;1:125. 
13. Ginsberg NA, Cohen L, Dungan JS, Concialdi S, Mangers $K$, Shulman LP. 3-D ultrasound of the fetal ear and fetal autosomal trisomies: a pilot study of a new screening protocol. Prenat Diagn 2011;31:311-4.

14. Crolla JA, Howard P, Mitchell C, Long FL, Dennis NR. A molecular and FISH approach to determining karyotype and phenotype correlations in six patients with supernumerary marker(22) chromosomes. Am J Med Genet 1997;72:440-7.
15. Knoll JH, Asamoah A, Pletcher BA, Wagstaff J. Interstitial duplication of proximal 22q: phenotypic overlap with cat eye syndrome. Am J Med Genet 1995;55:221-4.

16. Zhang J, Ma D, Wang Y, Cao L, Wu Y, Qiao F, et al. Analysis of chromosome 22q11 copy number variations by multiplex ligation-dependent probe amplification for prenatal diagnosis of congenital heart defect. Mol Cytogenet 2015;8:100. 